ARTICLE

Received 4 Dec 2014 | Accepted 19 May 2015 | Published 26 Jun 2015

DOI: $10.1038 /$ ncomms 8565

OPEN

\title{
Adiabatic elimination-based coupling control in densely packed subwavelength waveguides
}

Michael Mrejen ${ }^{1, \star}$, Haim Suchowski ${ }^{1, *}$, Taiki Hatakeyama ${ }^{1, \star}$, Chihhui $W^{1}{ }^{1}$, Liang Feng ${ }^{1}$, Kevin $^{\prime}$ Brien $^{1}$, Yuan Wang ${ }^{1} \&$ Xiang Zhang ${ }^{1,2}$

The ability to control light propagation in photonic integrated circuits is at the foundation of modern light-based communication. However, the inherent crosstalk in densely packed waveguides and the lack of robust control of the coupling are a major roadblock toward ultrahigh density photonic integrated circuits. As a result, the diffraction limit is often considered as the lower bound for ultra-dense silicon photonics circuits. Here we experimentally demonstrate an active control of the coupling between two closely packed waveguides via the interaction with a decoupled waveguide. This control scheme is analogous to the adiabatic elimination, a well-known procedure in atomic physics. This approach offers an attractive solution for ultra-dense integrated nanophotonics for light-based communications and integrated quantum computing.

\footnotetext{
${ }^{1}$ NSF Nanoscale Science and Engineering Center (NSEC), University of California, Berkeley 3112 Etcheverry Hall, UC Berkeley, California 94720, USA.

${ }^{2}$ Materials Sciences Division, Lawrence Berkeley National Laboratory, 1 Cyclotron Road, Berkeley, California 94720, USA. * These authors equally contributed to this work. Correspondence and requests for materials should be addressed to X.Z. (email: xiang@berkeley.edu).
} 
$\mathrm{T}$ he ever-growing need for energy-efficient and fast communications is driving the development of highly integrated photonic circuits where controlling light at the nanoscale becomes the most critical aspect of information transfer ${ }^{1}$. Routing and coupling devices based on multimode interference ${ }^{2-4}$ and evanescent coupling 5,6 are important building blocks in these integrated photonics circuits and have been employed as optical modulators, mode converters and switches for high-speed communication ${ }^{7-9}$, data processing and integrated quantum operations ${ }^{10-11}$. However, active control over the coupling between closely packed waveguides is highly desirable and yet remains a critical barrier towards ultra small footprint devices. A general approach to achieve active control in waveguide systems is to exploit optical non-linearities enabled by a strong control pulse $e^{12-18}$. However, these devices suffer from the non-linear absorption induced by the intense control pulse as the signal and its control propagate in the same waveguide ${ }^{12,17,18}$. Here we experimentally demonstrate a unique scheme based on adiabatic elimination (AE) concept that effectively manipulates the coupling between densely packed waveguides. We demonstrate active coupling control between two closely packed waveguides by tuning the mode index of an in-between decoupled waveguide. In this control scheme, the non-linearity influences the refractive index of a dark mode and thus leaves the signal unaffected by the induced losses. Such a scheme is a promising candidate for ultra-dense integrated nanophotonics such as on-chip ultrafast modulators and tunable filters for optical communication and quantum computing.

Two-coupled modes are a cornerstone in many research fields-from the dynamics of spin-half systems in nuclear magnetic resonances through two-level systems in atomic and molecular physics to polarization optics and directional couplers $6,19,20$. The dynamics in these systems are generally dictated by two independent quantities-the coupling strength and the phase difference between the interacting states or modes.
While active control of these quantities is available in the atomic systems, it remains a challenge in photonics realizations. Quantum control theory offers the mathematical framework to decompose multi-levels systems represented by multidimensional Hilbert spaces into controllable two-coupled mode systems ${ }^{21,22}$. For instance, in three-level atomic systems, several schemes were proposed to reduce the full eight-dimensional Hilbert space into the three-dimensional space of atomic two-level systems ${ }^{21-23}$. One example is the atomic stimulated rapid adiabatic passage (STIRAP) scheme ${ }^{24}$, which was recently shown in coupled waveguide systems ${ }^{25-28}$. However, this scheme cannot provide a dynamic control of energy transfer between the two outer waveguides, and moreover requires long propagation distance along the waveguides, which makes device scaling down difficult.

Here we propose and experimentally demonstrate a unique scheme to actively control the coupling among waveguides for densely integrated photonics based on AE. Analogous to atomic systems $\mathrm{s}^{24,29,30}, \mathrm{AE}$ is achieved by applying a decomposition on a three-waveguide coupler, where the two outer waveguides serve as an effective two-mode system analogous to the ground and excited states in an atomic three-level system, and the middle waveguide is the equivalent to the intermediate 'dark' state. We experimentally show the $\mathrm{AE}$ decomposition scheme in nanowaveguides and its capability of active control of the coupling between two waveguides by manipulating the mode index of a decoupled middle one. As the AE procedure separates the signal (information) from the control, it reduces significantly two-photon absorption (TPA) and TPA-induced free carrier absorption experienced by the signal, which are major obstacles in silicon photonics ${ }^{17,18}$. In addition, our analysis also includes the inherent higher order coupling, which is significant when packing is subwavelength, allowing control capabilities beyond what can be achieved in atomic physics. This new class of AE-based nanophotonic devices lays down the foundation for ultra-dense integrated photonic circuits.

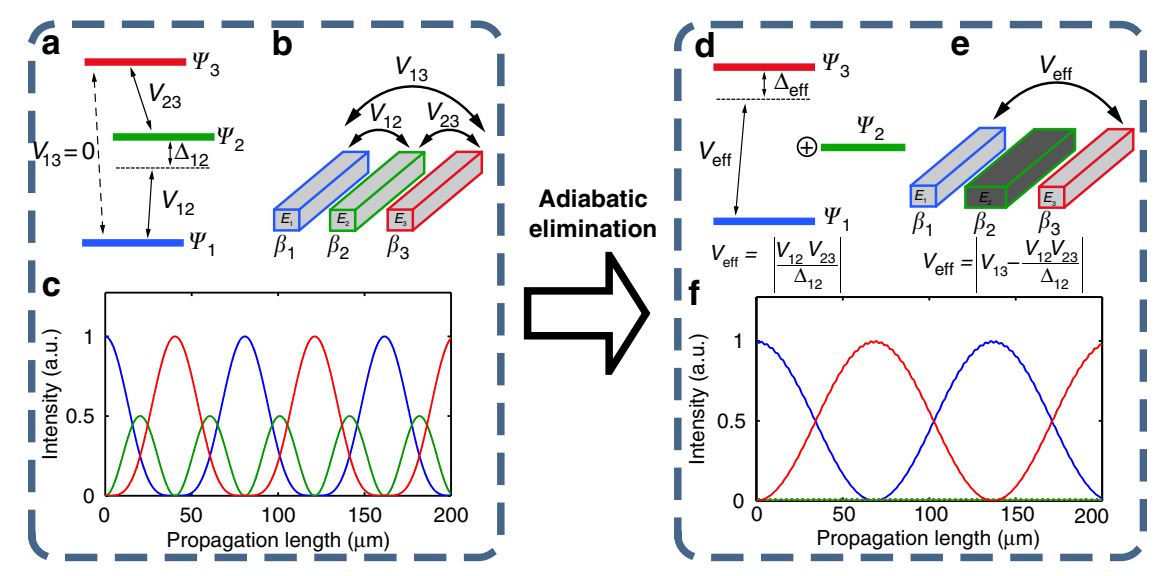

Figure 1 | AE scheme in atomic physics and optical waveguides. A general three-coupled mode system can be realized in (a) atomic systems and in (b) three-waveguide coupler. These systems share equivalent dynamics where the time evolution of the population of electrons in each level is analogous to the electric field propagation in each waveguide. In both cases, the evolution is dictated by the couplings between the modes, $V_{i j}$ and by the detunings $\Delta_{i j}$ or the difference between propagation constants $\Delta \beta_{i j}$. (c) The intensity evolution in three identical waveguides, where $V_{12}=V_{23}$ and the light is injected in waveguide 1. This evolution is equivalent to on-resonant three-level atomic interactions, where all the electrons are initially in the ground state. As seen, all the waveguides have a significant light intensity throughout the propagation as a result of the couplings. (d) AE process in the atomic system relies on a strong coupling between nearby levels that exhibit a very large detuning between them $\left(\Delta_{12}, \Delta_{23} \gg V_{12}, V_{23}\right)$. Since each of the two coupling processes is greatly detuned, the amplitude of the intermediate level oscillates very rapidly in comparison to the slow varying population in the other levels resulting in no significant build up and remaining at its initial value. The three-level system is thus reduced to an effective two-level system with an effective coupling $V_{\text {eff }}=\left|\frac{V_{12} V_{23}}{\Delta \beta_{12}}\right|$ between the ground and the excited states, with the intermediate level being a 'dark' state. (e) AE analogue in optical waveguides. The outer waveguides become an effective two-mode coupler with $V_{\text {eff }}=V_{13}-\frac{V_{12} V_{23}}{\Delta \beta_{12}}$ and the middle waveguide is a 'dark mode'. Importantly, the coupling between the two outer waveguides is now controllable through $\Delta \beta_{12}$. (f) When $A E$ conditions are met, the light injected in waveguide 1 propagates only in the outer waveguides 1 and 3. The middle waveguide is effectively eliminated, as its energy build-up remains very low during the entire propagation. 


\section{Results}

AE in a three-waveguide system. The evolution of the electrical fields in three-coupled mode systems can be described by the general equations ${ }^{31,32}: \quad \partial_{z} A_{i}=i\left(\Delta \beta_{i j} A_{i}+\sum_{j \neq i} V_{i j} A_{j}\right)$, $i, j=1,2,3$, where $A_{i}$ are the electromagnetic fields of the different modes, $V_{i j}$ is the coupling strength between waveguides $i$ and $j$ and $\Delta \beta_{i j}$ is the mode propagation constant difference, $\Delta \beta_{i j}=\beta_{i}-\beta_{j}=\frac{\omega}{c}\left(n_{i}^{\text {eff }}-n_{j}^{\text {eff }}\right)$, where $\omega$ is the frequency of the light, $c$ is the speed of light and $n_{i}^{\text {eff }}$ is the effective mode index of waveguide $i$. The AE procedure in waveguides, in analogy to atomic physics, relies on a strong coupling between nearby waveguides that exhibit a very large mode index mismatch between them $\left(\left|\Delta \beta_{12}\right|,\left|\Delta \beta_{23}\right| \gg V_{12}, V_{23}\right)$. Since each of the two consecutive coupling processes is greatly mismatched, the amplitude of the middle waveguide, $A_{2}$ will oscillate very rapidly in comparison to the slow varying amplitudes $A_{1}$ and $A_{3}$. As a result, the amplitude of the intermediate waveguide cannot build up significantly and thus remains as in itsitial value (that is, $\left|A_{2}(z)\right| \approx\left|A_{2}(z=0)\right|$. Figure 1 shows a schematic comparison between the evolution of identical three-mode system and the $\mathrm{AE}$ evolution both in the atomic physics realization and in directional waveguides.

Once AE conditions have been imposed, the effective coupling coefficient in this reduced two-level dynamics between the two outer waveguides becomes $V_{\text {eff }}=$ $\sqrt{\left(V_{13}^{*}+\frac{V_{23}^{*} V_{12}^{*}}{\Delta \beta_{12}}\right)\left(V_{13}-\frac{V_{12} V_{23}}{\Delta \beta_{23}}\right)}$, and energy can be efficiently transferred between the outer waveguides via the middle one when the outer waveguides have the same mode index $\left(\left|\Delta \beta_{12}+\Delta \beta_{23}\right| z \ll 1\right)$. This expression reveals that the coupling between the outer waveguides depends not only on the couplings in the original three-waveguide system, but also on the difference in the mode propagation constants between the outer waveguides and the middle waveguide even though the latter is 'eliminated'. In other words, one can control the coupling between the outer waveguides if the $\mathrm{AE}$ conditions can be met, for example, by changing the effective refractive index of the middle waveguide. Moreover, the efficiency of conversion from $A_{1}$ and $A_{3}$ does not
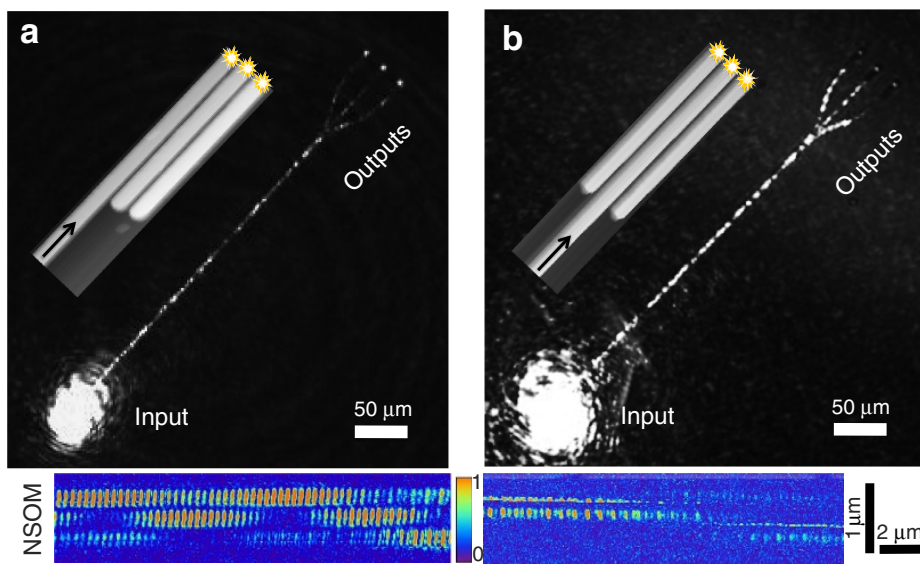

Identical waveguides
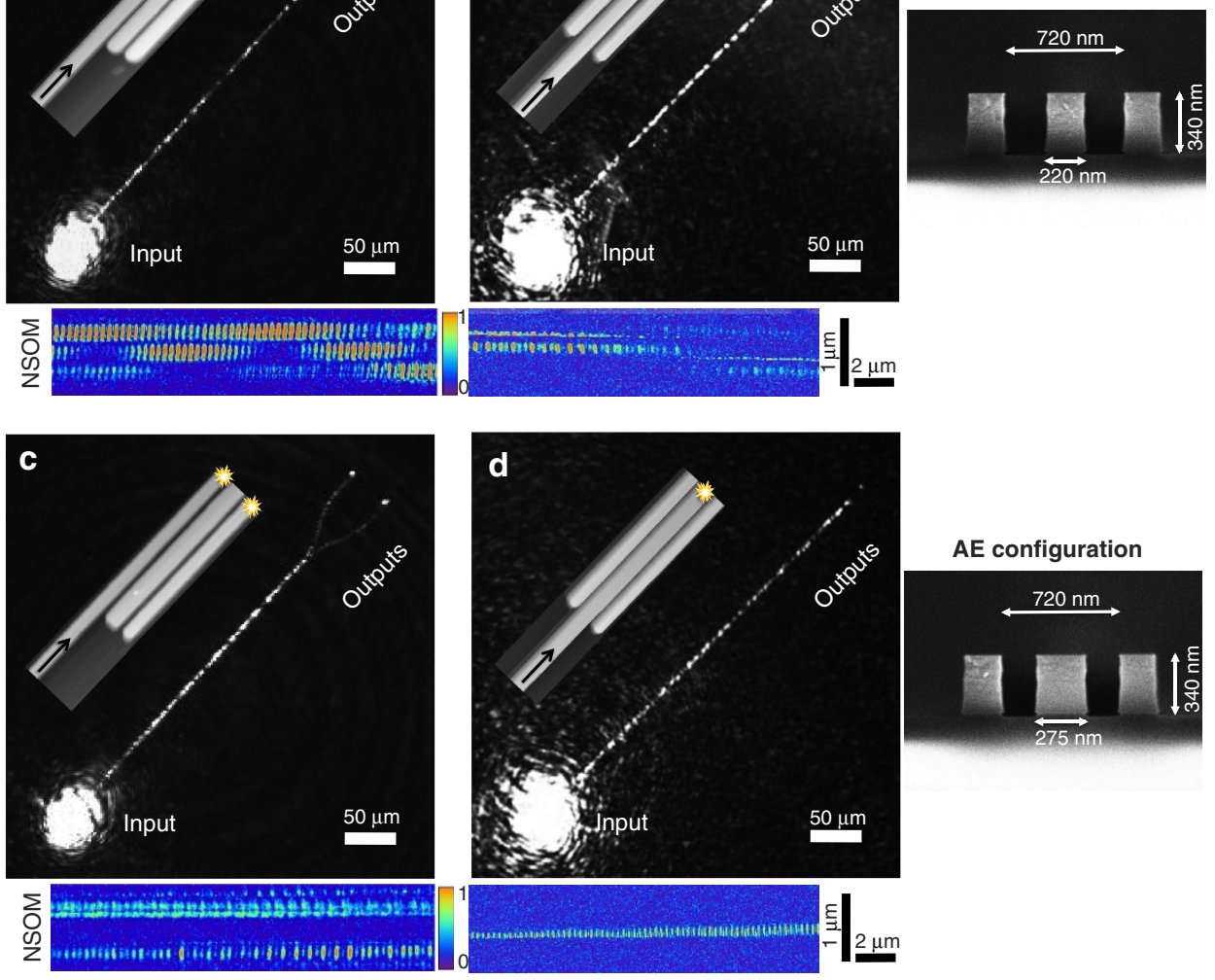

Figure 2 | Experimental observation of AE compared with ordinary three identical waveguides. (a,b) Far-field and NSOM measurements in three identical waveguides configuration. Owing to the coupling between the waveguides, light appears in all waveguides along the propagation, regardless if the input of the light is injected to $(\mathbf{a})$ the outer or $(\mathbf{b})$ the middle waveguide. (c,d) Experimental observation of $A E$ in an $A E$ configuration with a wider middle waveguide. We observe the ' $2+1$ ' decomposition of three-coupled modes into (c) an effective two-coupled mode system of the outer waveguides and (d) a dark middle waveguide. (c) Light is coupled to an outer waveguide in an AE configuration. Far-field image shows that only the outer waveguides emit light at the output. The near-field image confirms the absence of light in the middle waveguide along the entire propagation. A full NSOM scan showing a complete inversion can be viewed in Supplementary Fig. 8. (d) Light is coupled to the middle waveguide in an AE configuration. Only the middle waveguide emits light at the output without coupling into nearby outer waveguides. The near-field image shows that no light leaks out from the middle waveguide. Atomic force microscopy images (inset) show each of the three waveguides with the inputs and the outputs. The scanning electron microscopy images (right column) show the cross-section of the fabricated waveguides for the AE configuration and for the identical waveguides configuration. We used a CW laser with $\lambda=1,310 \mathrm{~nm}$, the width of the outer waveguides was fixed to $W_{1}=W_{3}=220 \mathrm{~nm}$, where for the $A E$ configuration $W_{2}=275 \mathrm{~nm}$, and for the control waveguide configuration $W_{2}=220 \mathrm{~nm}$. The height of the waveguides is $340 \mathrm{~nm}$ and the gap between the outer waveguides kept at $720 \mathrm{~nm}$ for both cases. The propagation length is $300 \mu \mathrm{m}$ for all configurations. Colour bars for the NSOM measurements are for the intensity and are in arbitrary units. 

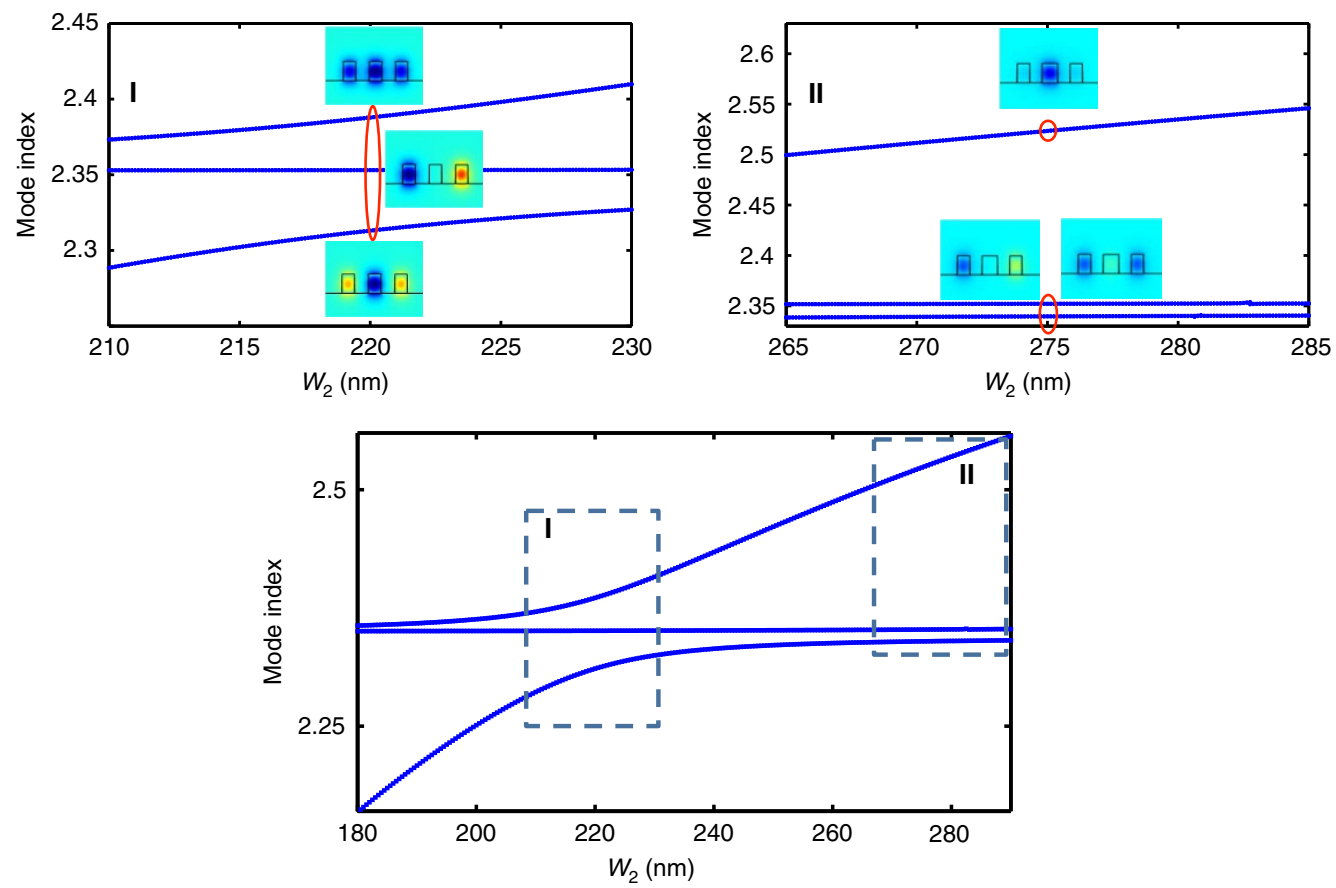

Figure 3 | Band diagram of a three-mode coupler with varying middle waveguide width $\boldsymbol{W}_{\mathbf{2}}$. The band diagram shows the electric field eigenvalues (blue curves) and eigenmodes (colour maps) of the system for TM polarization (for TE polarization, see Supplementary Fig. 4). (Inset I) All the three waveguides are identical, $W_{1}=W_{2}=W_{3}=220 \mathrm{~nm}$ and are mixed in the eigenmodes of the system. In this configuration, an input in one of the waveguides is projected necessarily on all the waveguides. (Inset II) AE regime. The AE is reached when $W_{2}$ is larger and our sample (red circles, $W_{2}=275 \mathrm{~nm}$ ) is in this regime. Here an eigenmode involving only the middle waveguide emerges and becomes less coupled, since the coupling is proportional to $1 / \Delta n$ where $\Delta n=n_{1}^{\text {eff }}-n_{2}^{\text {eff }}$, leading to the elimination of the middle waveguide. The two remaining eigenmodes are symmetric and antisymmetric superpositions of the outer waveguides as in a conventional two-mode coherent coupler. An input in either of the outer waveguides will be projected only onto those two eigenmodes, resulting in an effective two-waveguide system evolution, while the middle waveguide remains 'dark' along the propagation. On the other hand, an input injected in the middle waveguide is projected only onto the dark mode of the system and will stay 'trapped' in the middle waveguide without transferring energy to the adjacent ones.

change even when the middle waveguide is extremely lossy as we show in the Supplementary Discussion 2. An important parameter that characterizes the degree of elimination in such an adiabatic process is $\frac{V_{12}}{\Delta \beta_{12}}$, which should be much lower than unity to ensure AE evolution. Also, it is worth noting that the propagation constant mismatch of the effective two-mode system is modified as well: $\Delta \beta_{\text {eff }}=\Delta \beta_{12}+\Delta \beta_{23}+\frac{\left|V_{12}\right|^{2}}{2 \Delta \beta_{12}}+\frac{\left|V_{23}\right|^{2}}{2 \Delta \beta_{23}}=$ $\Delta \beta_{\mathrm{TP}}+\Delta \beta_{s}$, where in addition to the conventional phasemismatch terms, two more terms, equivalent to the Stark-shift terms in atomic physics, influence the evolution. This atomic physics analogue for active control with a nanoscale footprint expands earlier theoretical analyses of three-waveguide couplers $^{33-35}$, and brings new insight for subwavelength silicon devices. It should also be noted that in contrast to the waveguide analogue to STIRAP, where the couplings between the waveguides vary adiabatically along the propagation, in the $\mathrm{AE}$ scheme the couplings between the waveguides remain fixed, and the adiabaticity resides in the slow oscillating energy transfer between waveguide 1 and 3 as compared with the very fast oscillations between 1 and 2 and 2 and 3 .

Observation of the $\mathrm{AE} 2+\mathbf{1}$ decomposition in waveguides. We experimentally demonstrate this unique $\mathrm{AE}$ in directional waveguides using silicon-on-insulator (SOI) platform. The sample consists of three waveguides, where two identical outer waveguides have the same width, thus the same effective refractive index and a varying middle waveguide width. The middle waveguide was designed wider than the outer waveguides to meet the AE conditions, while using same width for the control sample. In all the configurations, the gap between the outer waveguides was kept constant.

We observed that in the AE configuration, the dynamics is indeed decomposed into an effective two-mode system and a dark mode. When light was coupled to an outer waveguide (\#1), it remains only in the two outer waveguides along the entire propagation (Fig. 2c). On the other hand, when light is injected to the middle waveguide, it remains trapped along the propagation, without coupling to the outer waveguides (Fig. 2d). In all cases, properly designed grating couplers and spot size converters have been employed. This is in clear contrast to the controls where all the waveguides participate in the evolution for both injections Fig. 2a,b. Also, we have confirmed these dynamics with both far-field and near-field measurements. Those are in a very good agreement with the numerical simulation presented in Supplementary Fig. 8.

Band diagram picture of the $\mathrm{AE}$ in waveguides. The $\mathrm{AE}$ process in three-coupled waveguides system can be understood using a band diagram of the system's eigenmodes as a function of the middle waveguide width $W_{2}$ (Fig. 3). We observe that in the control configuration the eigenmodes of the coupled system involve necessarily all of the waveguides. However, the $\mathrm{AE}$ regime, which occurs when the mode index of the middle waveguide differs significantly from the mode index of the outer waveguides, gives rise to an increasingly decoupled eigenmode involving the middle waveguide only. The two other eigenmodes involve only the outer waveguides in a symmetric and anti- 

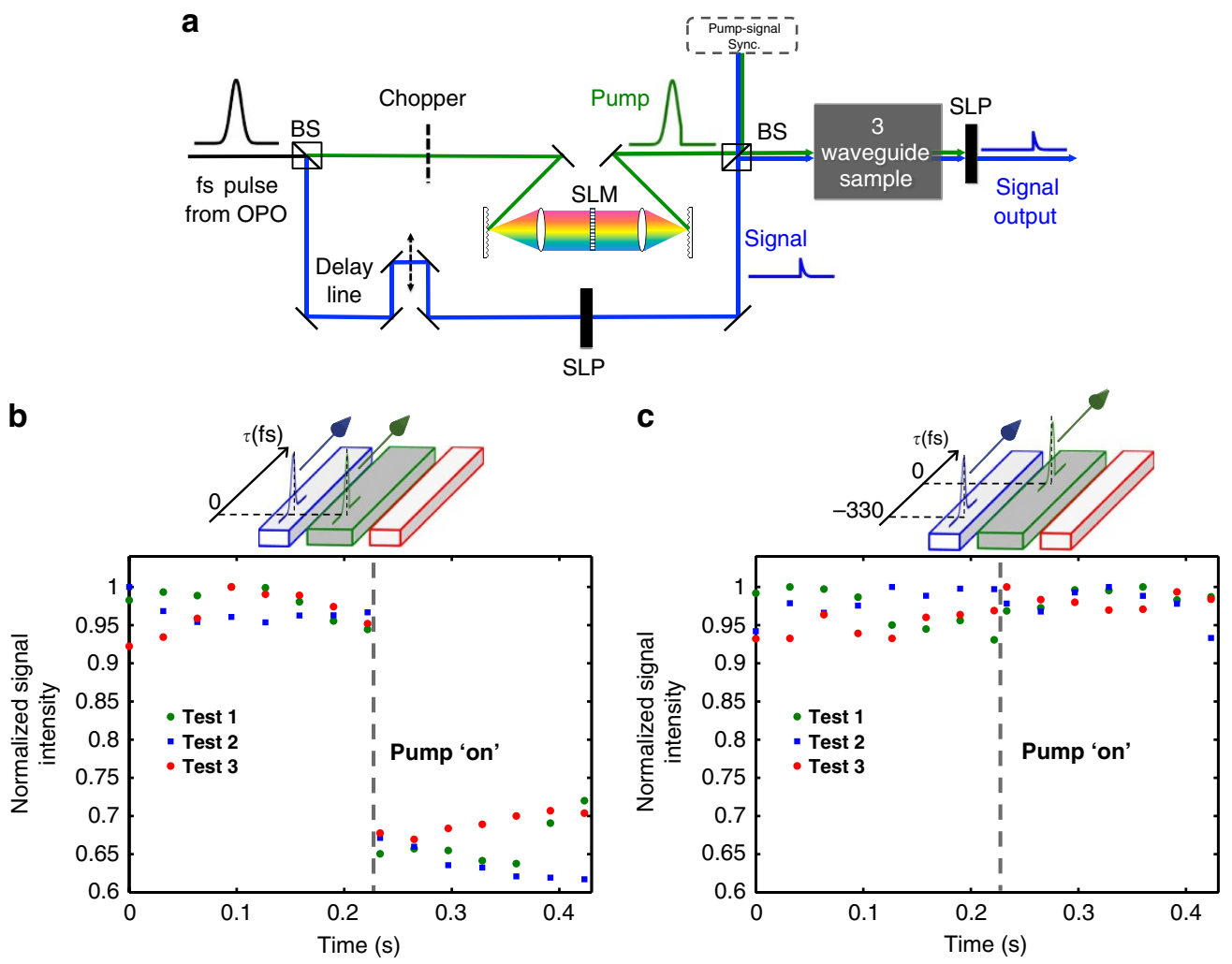

Figure 4 | Active coupling control between outer waveguides in AE configuration. (a) Schematic of the experimental apparatus. The ultrashort pulse ( $\sim 140 \mathrm{fs}$ with central wavelength at 1,310 $\mathrm{nm}$ ) is split into a strong truncated pump beam that is coupled to the middle waveguide and the weak signal beam that is coupled to one of the outer waveguide. On the pump path, a mechanical chopper is used to turn off and on the injection of the pump pulse to the middle waveguide. On the signal path, a delay line is used to synchronize the entrance of the pump and signal. A full description of the experimental procedure is provided in the Supplementary Method 2 (b) Observed modulation as a result of varied coupling in the presence of a synchronized pump beam, showing a significant reduction of the signal intensity when the pump is present. (c) In the presence of a delayed pump beam ( $330 \mathrm{fs}$ delay between pump and signal), no change in the signal output is observed. This indicates that the modulation of index originates from the fast Kerr non-linearity rather than long-lived free carriers (FC) effect. A discussion regarding the FC role in this experiment is provided in the Supplementary Discussion 7. Several tests are performed to show repeatability of the process and exclude laser fluctuations (shown here as different coloured data points in $\mathbf{b}, \mathbf{c}$ ).

symmetric fashion, similar to the conventional two-mode coherent coupler. Hence, in the AE regime, the dynamics can be split into $2+1$ (effective two-level and a 'dark state', respectively) dynamical spaces. This decomposition occurs for both TM (shown in Fig. 3) and TE polarizations (see Supplementary Fig. 4). Moreover, the AE regime is very robust and broadband (see Supplementary Discussions 4 and 5). Also, we note that in the case where the middle waveguide is narrower than the outer waveguide, that is, its mode index is smaller than the outer modes indices, another regime of $\mathrm{AE}$ dynamics exists with possible higher effective couplings (as the two contributions in the effective coupling expression will add up constructively); however, with a risk of wavelength cutoff.

Active coupling control based on AE. We now apply the AE scheme to dynamically control the effective coupling between the outer waveguides by changing the mode index of the decoupled middle waveguide. Taking advantage of the large nonlinear Kerr coefficient of silicon ${ }^{36}$ combined with the high light confinement enabled by the subwavelength cross-section of Silicon photonic waveguides $^{37,38}$, we obtained a localized index change in the middle waveguide.

Using a single-shot non-linear technique ${ }^{39}$, we couple the signal to one of the outer waveguides, while the pump beam is coupled to the middle waveguide (Fig. 4a). The AE evolution confines the pump beam to the middle waveguide along the propagation thus ensuring no leakage to the outer waveguides. We insure proper synchronization between the signal and the pump using the procedure described in the Supplementary Method 1 and in the Supplementary Fig. 9. We clearly observe that the signal collected is modulated in the presence of the pump (Fig. 4b). The modulation originates from the non-linear change of refractive index in the middle waveguide as the pump beam propagates. This change, which increases the phasemismatch between the middle and the outer waveguides $\left(n_{\text {middle }}=n_{\text {middle }}^{0}+n_{2} I_{\text {pump }} \rightarrow \Delta \beta_{12}=\Delta \beta_{12}^{0}+2 \pi n_{2} I_{\text {pump }} / \lambda\right), \quad$ in turn affects the effective coupling between waveguides \#1 and \#3 which, finally, leads to a change in the inversion length $\left(L_{\mathrm{inv}}\right)$, manifested as a change of output intensity in a waveguide of a given length ( $\Delta \beta_{12}$ increases $\rightarrow V_{\text {eff }}$ decreases $\rightarrow L_{\text {inv }}$ increases).

Our numerical simulations of the coupled non-linear Schrödinger equations show that this result discussed above is consistent with a change of index of $\Delta n=(7.15 \pm 0.25) \times 10^{-3}$ in the middle waveguide with a pulse energy of $66 \pm 6 \mathrm{pJ}$. We also confirm that in the presence of the pump in the middle waveguide the inversion length in the outer waveguides increases. We have checked the effect as a function of pump-signal delay and confirmed that the effect observed in the synchronized measurements stems from an ultrafast change of index in the middle waveguide and that TPA-generated free carriers do no play a significant role in the process (Fig. 4c) $)^{17,18}$

As we have demonstrated, this unique scheme separates spatially the control in the middle waveguide from the signal in 
the outer waveguides. Therefore, other physical effects can be employed to change the mode index of the middle waveguide such as thermal, mechanical or electronic processes. More importantly, as we show in the Supplementary Discussion 2, the AE scheme holds also when the middle waveguide is lossy. The loss originates from the imaginary part of the refractive index, which can be either due to a linear effect, as in plasmonic and near resonant interactions, or a non-linear effect, where imaginary part of a third-order susceptibility is intensity dependent and alters the index of refraction, as in TPA- and FCA-induced losses in silicon. In such cases, the effective mode index of the middle waveguide as well as the coupling coefficients will become complex valued. Nonetheless, when employing the $\mathrm{AE}$ procedure, which equalizes the rate of light transfer to and from the middle waveguide, the effective propagation length of the signal in a lossy middle waveguide is minimized; thus, the effective loss in the effective two-waveguides evolution will be significantly reduced as compared with the equivalent twowaveguide system (see Supplementary Figs 2 and 3 ). The reduced effective loss in the $\mathrm{AE}$ configuration holds the potential to further reduce the footprint with plasmonic waveguides without facing the losses typically associated ${ }^{40,41}$.

Inspired by the atomic $\mathrm{AE}$, our scheme of active control for nanoscale waveguiding brings new insight for subwavelength silicon photonics. Since AE enables unprecedented tunable coupling, it further allows a zero effective coupling between the outer waveguides that cancels light transfer and eliminates the crosstalks between waveguides, a must for ultra-dense nanophotonics interconnects. This is due to a degeneracy in the coupled system, where the sequential coupling strength $\left(V_{12} V_{23} / \Delta \beta\right)$ is equal to the direct coupling $\left(V_{13}\right)$, leading to a full destructive interference along the entire propagation. This degeneracy happens when the middle waveguide's effective index is higher than the outer waveguides, that is, when $\Delta \beta=\Delta \beta_{23}=-\Delta \beta_{12}>0$. We found that this constraint can be satisfied for TE mode where the sizes of the waveguides are of the same order as the gap between the waveguides, thus allowing significant coupling between the outer waveguides. Hence, at this singular point, all three waveguides are decoupled from each other despite the fact they are densely packed with a subwavelength gap (see Supplementary Fig. 1), thus yielding the capability to shield information from the surrounding within the evanescent range. Therefore, $\mathrm{AE}$ scheme provides a new way in achieving dense optical waveguiding with negligible crosstalk. For example, for a given gap between two waveguides of $800 \mathrm{~nm}$, coupling length under AE conditions can be as long as $1.3 \mathrm{~mm}$, in sharp contrast with $105 \mu \mathrm{m}$ in a conventional directional coupler with the same gap. However, according to our simulations, dimensional accuracy required for the realization of such excellent shielding is on the order of a few nanometres, a challenging fabrication task at present. It is nonetheless possible as the nanofabrication further improves.

\section{Discussion}

In conclusion, we have experimentally demonstrated the $\mathrm{AE}$ decomposition scheme in nanowaveguides. We have shown that this approach enables on demand dynamical control of the coupling between two closely packed waveguides, by modulating the mode index of an in-between decoupled waveguide. This is in contrast to the conventional directional couplers where the constant coupling coefficient is predetermined by the distance in between the waveguides. AE offers an attractive alternative route for the control of optical information in integrated nanophotonics. The AE approach provides a new flexible toolbox to design densely packed power-efficient nanoscale photonic components, such as compact modulators, ultrafast optical signal routers and interconnects.

\section{Methods}

Numerical simulations. The design of the samples was carried out using semi-analytical calculations and COMSOL multiphysics simulations aimed to examine the generality of the AE scheme. We have also studied the dynamics in the modulation experiment by performing numerical simulation of the coupled non-linear Schrödinger equations using finite difference calculation. In our simulations, we have used the non-linear parameters of silicon ${ }^{36}$ $\left(n_{\mathrm{kerr}}=1.7 \times 10^{-5} \mathrm{~cm}^{2} \mathrm{GW}^{-1}\right)$ and estimated the pump intensity in the middle waveguide to be $\left(I_{\text {pump }}=430 \mathrm{GW} \mathrm{cm}^{-2}\right)$.

Sample preparation. Silicon waveguides were fabricated in a silicon-on-insulator (SOI) substrate. The thickness of silicon and buried oxide were $340 \mathrm{~nm}$ and $1 \mu \mathrm{m}$, respectively. A 160-nm-thick hydrogen silsesquioxane (HSQ) resist (Dow Corning XR-1541) was spun on the SOI substrate. The HSQ layer was patterned by electron-beam lithography for the etching mask. The silicon waveguides were formed by reactive ion etching in $\mathrm{Cl} 2 / \mathrm{HBr} / \mathrm{O} 2$. 10:1 buffered $\mathrm{HF}$ was used to remove the HSQ mask.

The light propagation in the waveguides was examined both with far-field and near-field set-ups described below.

Far-field measurements. The far-field measurements, presented in Fig. 2, were carried out on microscope with a $\times 10 /$ NA 0.3 objective in reflection mode. The light from a CW laser diode at $1,310 \mathrm{~nm}$ is focused on the input grating. We collect the output light from the waveguides in reflection and image them an uncooled infrared focal plane array (IR FPA, Goodrich). The waveguides roughness scatters the coupled light along the propagation and serves as an indication of proper coupling ${ }^{42}$.

Near-field scanning optical microscopy measurements. We acquired the near-field scanning optical microscopy (NSOM) images in Fig. 2 and in the Supplementary Fig. 8 using a home-built scattering near-field optical microscopy $(\mathrm{s}-\mathrm{NSOM})^{43}$ in a transmission configuration. In this set-up, light from a CW laser diode at $1,310 \mathrm{~nm}$ is focused on the input grating using a $\times 10 / \mathrm{NA} 0.3$ objective. To ease the alignments, we used a PtIr coated Advanced Tip at the End of Cantilever (ATEC) with a tip radius $<10 \mathrm{~nm}$ and tapping frequency of $\Omega \sim 300 \mathrm{kHz}$. The ATEC was moved along the waveguides with subnanometre precision using piezostages to record the propagation of the input light. Light scattered from and modulated by the tip was collected using an aspheric lens $(\mathrm{NA}=0.16)$ and focused onto a $10-\mathrm{MHz}$ InGaAs photoreceiver (NewFocus 2053 FS). The signal was demodulated at $3 \Omega$ to filter out the non-near-field background scattering.

Ultrafast modulation measurements. The ultrafast experimental set-up, shown schematically in Fig. 4a, is based on a variant of the single-shot non-linear technique $^{39}$ where an ultrashort pulse from a Ti:Sa pumped OPO (Coherent/APE Chameleon Compact OPO, repetition rate $80 \mathrm{Mhz}, 140 \mathrm{fs}$ ) centred at $1,310 \mathrm{~nm}$ is split into strong pump beam ( $70 \mathrm{pJ}$ energy per pulse) that is coupled to the middle waveguide and weak signal beam that is coupled to one of the outer waveguide (for convenience, we note it as waveguide \#1). To ensure that the signal measured only arises from the light coupled to the outer waveguide, we spectrally truncate at $1,319 \mathrm{~nm}$ the red tail of the pump beam using a pulse shaper. We cut the tail in a way that the time envelope is minimally perturbed. We estimate the final pulse width to be no more than $200 \mathrm{fs}$. The truncated pump beam is coupled to the middle waveguide with a grating coupler. The signal beam is passed through a sharp longpass filter (Semrock $1319 \mathrm{~nm}$ RazorEdge ultrasteep longpass edge filter) before being injected waveguide \#1 in a similar fashion. As the evolution in the AE configuration confines the pump beam to the middle waveguide along the propagation, it prevents leakage to the outer waveguides. The method used to ensure that the pump and signal beams are coupled in the waveguides at the exact same time (with an accuracy of $4 \mathrm{fs}$ ) is described in the Supplementary Method 1. The light propagated in the outer waveguide is out-coupled to the free space and imaged on an uncooled IR FPA (Goodrich), after passing through a sharp longpass filter (Semrock 1,319 nm RazorEdge ultrasteep longpass edge filter). The wavelengths contributing to the image formed on the camera are spectrally located in the tail that has been cut away from the pump but left in the signal. With a mechanical chopper, we turn on and off the pump beam while recording the images from the IR FPA at 31 frames per second. We processed the acquired images to extract the output from the signal waveguide as a function of the pump.

\section{References}

1. Vlasov, Y. A. Silicon integrated nanophotonics: road from scientific explorations to practical applications. Plenary talk, CLEO. Available at http://researcher.watson.ibm.com/researcher/files/us-yvlasov/vlasov_CLEO_ Plenary_05092012.pdf (2012). 
2. Soldano, L. B. \& Pennings, E. C. M. Optical multi-mode interference devices based on self-imaging: principles and applications. J. Lightwave Technol. 13, 615-627 (1995).

3. Kawaguchi, Y. \& Tsutsumi, K. Mode multiplexing and demultiplexing devices using multimode couplers. Electron. Lett. 38, 1701-1702 (2002).

4. Huang, Y., Xu, G. \& Ho, S.-T. An ultracompact optical mode order converter. Photon. Technol. Lett. 18, 2281-2283 (2006).

5. Luo, L.-W. et al. WDM-compatible mode-division multiplexing on a silicon chip. Nat. Commun. 5, 3069 (2014).

6. Yariv, A. Coupled-mode theory for guided-wave optics. IEEE J. Quantum Electron. 9, 919-933 (1973).

7. Green, W. M., Rooks, M. J., Sekaric, L. \& Vlasov, Y. A. Ultra-compact, low RF power, $10 \mathrm{~Gb} / \mathrm{s}$ silicon Mach-Zehnder modulator. Opt. Express 15, 17106-17113 (2007).

8. Xu, Q., Schmidt, B., Pradhan, S. \& Lipson, M. Micrometre-scale silicon electro-optic modulator. Nature 435, 325-327 (2005).

9. Reed, G. T., Mashanovich, G., Gardes, F. Y. \& Thomson, D. J. Silicon optical modulators. Nat. Photonics 4, 518-526 (2010).

10. Peruzzo, A. et al. Quantum walks of correlated photons. Science 329, 1500-1503 (2010).

11. Peruzzo, A. et al. Quantum delayed-choice experiment. Science 338, 634-637 (2012).

12. Almeida, V. R., Barrios, C. A., Panepucci, R. R. \& Lipson, M. All-optical control of light on a silicon chip. Nature 431, 1081-1084 (2004).

13. Jensen, S. M. The nonlinear coherent coupler. IEEE J. Quantum Electron. 18, 1580-1583 (1982).

14. Friberg, S. R., Weiner, A. M., Silberberg, Y., Sfez, B. G. \& Smith, P. S. Femtosecond switching in a dual-core-fiber nonlinear coupler. Opt. Lett. 13, 904-906 (1988).

15. Stegeman, G. I. \& Seaton, C. Nonlinear integrated optics. J. Appl. Phys. 58, R57 (1985).

16. Lederer, F. et al. Discrete solitons in optics. Phys. Rep. 463, 1-126 (2008).

17. Boyraz, Ö., Koonath, P., Raghunathan, V. \& Jalali, B. All optical switching and continuum generation in silicon waveguides. Opt. Express 12, 4094-4102 (2004).

18. Villeneuve, A. et al. Nonlinear absorption in a GaAs waveguide just above half the band gap. IEEE J. Quantum Electron. 30, 1172-1175 (1994).

19. Simon, R. U. A. Polarization optics of twisted single-mode fibers. Appl. Optics 18, 2241-2251 (1979).

20. Allen, L. \& Eberly, J.H. Optical resonance and two-level atoms (Courier Dover Publications, 1975).

21. D'Alessandro, D. Introduction to Quantum Control and Dynamics (CRC, 2007).

22. Polack, T., Suchowski, H. \& Tannor, D.J. Uncontrollable quantum systems: a classification scheme based on Lie subalgebras. Phys. Rev. A 79, 053403 (2009).

23. Shore, B. W. \& Cook, R. J. Coherent dynamics of N-level atoms and molecules. IV. Two- and three-level behavior. Phys. Rev. A 20, 1958-1964 (1979).

24. Vitanov, N. V., Halfmann, T., Shore, B. W. \& Bergmann, K. Laser-induced population transfer by adiabatic passage techniques. Annu. Rev. Phys. Chem. 52, 763-809 (2001).

25. Lahini, Y., Pozzi, F., Sorel, M., Morandotti, R. \& Silberberg, Y. EIT and STIRAP in Waveguides: Linear and Nonlinear Effects in a Three-Core Coupled System. in Bragg Gratings, Photosensitivity, and Poling in Glass Waveguides, OSA Technical Digest (CD) (Optical Society of America, 2007), paper JWBPDP1.

26. Lahini, Y. et al. Effect of nonlinearity on adiabatic evolution of light. Phys. Rev. Lett. 101, 193901 (2008).

27. Longhi, S. Adiabatic passage of light in coupled optical waveguides. Phys. Rev. E 73, 026607 (2006).

28. Ciret, C., Coda, V., Rangelov, A. A., Neshev, D. N. \& Montemezzani, G. Broadband adiabatic light transfer in optically induced waveguide arrays. Phys. Rev. A 87, 013806 (2013).

29. Tannor, D. Introduction to Quantum Mechanics: A Time-Dependent Perspective (University Science Book, 2006).
30. Brion, E., Pedersen, L. H. \& Mølmer, K. Adiabatic elimination in a lambda system. J. Phys. A: Math. Theor. 40, 1033-1043 (2007).

31. Shuang, S.L. Physics of Photonics Devices 2nd ed. 300-307 (Wiley, 2009).

32. Yariv, A. Optical Electronics 4th ed. 519-520 (Saunders College Publishing, 1991).

33. Hardy, A. \& Streifer, W. Coupled mode solutions of multiwaveguide systems. IEEE J. Quantum Electron. 22, 528-534 (1986).

34. Ruschin, S. \& Marom, E. Coupling effects in symmetrical three-guide structures. JOSA A 1, 1120-1128 (1984).

35. Kalonakis, K. \& Paspalakis, E. Optical switching in a symmetric three-waveguide nonlinear directional coupler. J. Modern Opt. 52, 1885-1892 (2005).

36. Lin, Q. et al. Dispersion of silicon nonlinearities in the near infrared region. Appl. Phys. Lett. 91, 021111 (2007).

37. Foster, M. A., Turner, A. C., Lipson, M. \& Gaeta, A. L. Nonlinear optics in photonic nanowires. Opt. Express 16, 1300-1320 (2008).

38. Leuthold, J., Koos, C. \& Freude, W. Nonlinear silicon photonics. Nat. Photonics 4, 535-544 (2010).

39. Dudovich, N., Oron, D. \& Silberberg, Y. Single-pulse coherently controlled nonlinear Raman spectroscopy and microscopy. Nature 418, 512-514 (2002).

40. Volker, J. et al. Experimental demonstration of low-loss optical waveguiding at deep sub-wavelength scales. Nature Communications 2, 331 (2011).

41. Oulton, R., Sorger, V., Genov, D. A., Pile, D. F. P. \& Zhang, X. A hybrid plasmonic waveguide for subwavelength confinement and long range propagation. Nature Photonics 2, 496-500 (2008).

42. Cooper, M. L. et al. Quantitative infrared imaging of silicon-on-insulator microring resonators. Opt. Lett. 35, 784-786 (2010).

43. Hillenbrand, R. \& Keilmann, F. Complex optical constants on a subwavelength scale. Phys. Rev. Lett. 85, 3029-3032 (2000).

\section{Acknowledgements}

This research was supported by the Office of Naval Research (ONR) MURI programme under Grant No. N00014-13-1-0678.

\section{Author contributions}

H.S., M.M. and C.W. performed the theoretical calculations; M.M. designed the samples T.H. and L.F. fabricated the samples; M.M., H.S. and K. O. conducted the experiments; M.M. and H.S. performed the data analysis. M.M., H.S., Y.W. and X.Z. prepared the manuscript. X.Z. guided the research. All authors contributed to discussions.

\section{Additional information}

Supplementary Information accompanies this paper at http://www.nature.com/ naturecommunications

Competing financial interests: The authors declare no competing financial interests.

Reprints and permission information is available online at http://npg.nature.com/ reprintsandpermissions/

How to cite this article: Mrejen, M. et al. Adiabatic elimination-based coupling control in densely packed subwavelength waveguides. Nat. Commun. 6:7565 doi: 10.1038/ncomms8565 (2015).

This work is licensed under a Creative Commons Attribution 4.0 International License. The images or other third party material in this article are included in the article's Creative Commons license, unless indicated otherwise in the credit line; if the material is not included under the Creative Commons license, users will need to obtain permission from the license holder to reproduce the material. To view a copy of this license, visit http://creativecommons.org/licenses/by/4.0/ 